\title{
Design and Matlab Simulation of Persian License Plate Recognition Using Neural Network and Image Filtering for Intelligent Transportation Systems
}

\author{
Omid Akbarzadeh $\oplus^{1}$, Mohammad R. Khosravi $\mathbb{(}^{2, *}$ and Linss T. Alex $\oplus^{3}$ \\ ${ }^{1}$ Department of Electronics and Telecommunications Politecnico di Torino, Turin, Italy; \\ omid.akbarzadeh@studenti.polito.it \\ ${ }^{2}$ Department of Computer Engineering Persian Gulf University, Bushehr, Iran; mohammadkhosravi@acm.org \\ ${ }^{3}$ Department of Electrical and Electronics Engineering (EEE) Albertian Institute of Science and Technology (AISAT), \\ Kochi 682022, Kerala, India; linsstalex@aisat.ac.in
}

\section{Academic Editor: \\ (1) Xin Ning \\ Institute of Semiconductors, \\ Chinese Academy of Sciences, \\ China}

Submitted: 23 Aug 2021

Accepted: 6 Oct 2021

Published: 8 Feb 2022

Vol. 2, No. 1, 2022.

do) $10.52810 /$ TPRIS. 2021.100098

*Correspondence:

Mohammad R. Khosravi

mohammadkhosravi@acm.org

\begin{abstract}
Vehicles play a vital role in modern-day intelligent transportation systems (ITS). Plate characters provide a standard means of identification for any vehicle. To serve this purpose, an automatic license plate recognition system is studied. In this paper, we intend to create an optimized algorithm for implementing the scheme. Firstly, we undertake several challenging stages. The first step is introduced as the determination of plate location. Then, in the second phase, we apply an initial improvement to decline the likely noises using the Gaussian function to provide an appropriate filter for this target. Next, the rest of the project is organized as follows, finding the edge of images, enhancing modified pictures, and selecting the exact place of the plate. Afterward, tilting and plate rotation improvement and plate characters' extraction are considered two essential steps in this regard. Eventually, the final step of this project consists of several stages, such as employing a neural network to extract the plate characters automatically.
\end{abstract}

Keywords: License Plate Recognition, Image Processing, Detection Algorithm, Probabilistic Neural Network

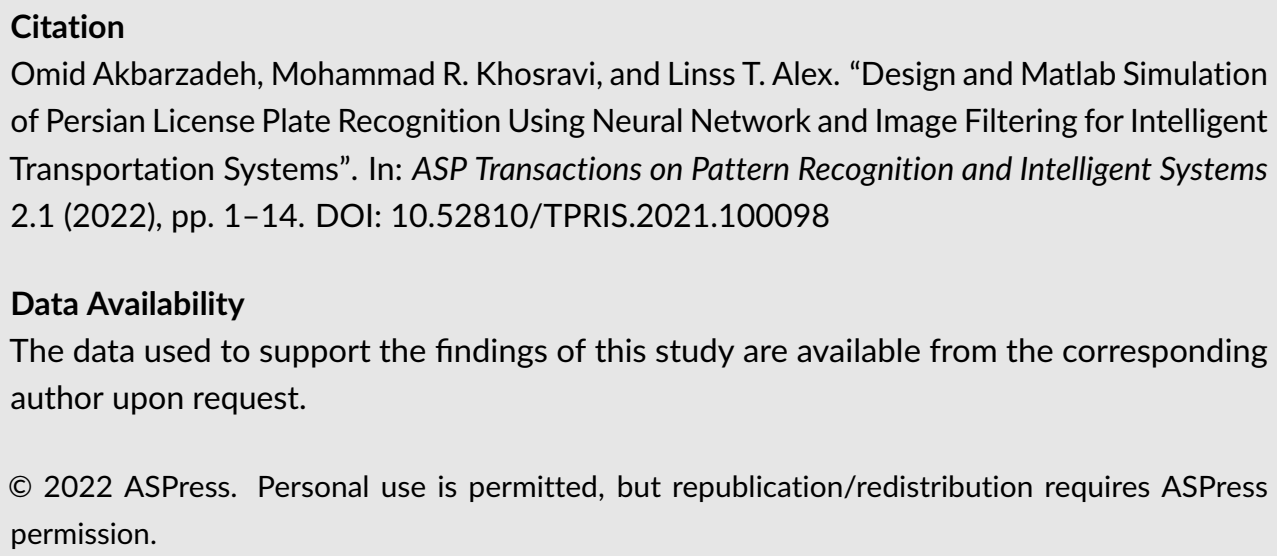
of Persian License Plate Recognition Using Neural Network and Image Filtering for Intelligent Transportation Systems". In: ASP Transactions on Pattern Recognition and Intelligent Systems 2.1 (2022), pp. 1-14. DOI: 10.52810/TPRIS.2021.100098

Data Availability

The data used to support the findings of this study are available from the corresponding author upon request.

(C) 2022 ASPress. Personal use is permitted, but republication/redistribution requires ASPress permission.

\section{Introduction}

Because the number of motor vehicles has risen drastically, it is evident that human resources could not manage and control traffic even on a small scale, 
such as small parking [1-3]. Apart from the fact that the cost of human resources is enormously high, its efficiency is lower than automated approaches. Hence an urgent need exists for mechanization in this field. In an automatic identification system of the license plate numbers, the primary purpose is to precisely identify the location of the plate in images to make a computer able to read the plate's characters [3-4]. In the foreseeable future, the automatic recognition of cars plate will be substituted with conventional methods.

This system will have a wide variety of use-cases in everyday life [5]. Since the license plate is unique for each car, there will be numerous different and practical applications for automated plate detection. As an illustration, implementing the intelligent parking according to this technology, the entrance gate will be opened and closed just for authorized cars; additionally, in vehicles, speed control operations and management of vehicles through the tolls [6-7]. This technology is practical for areas that need constant observation and an electronic penalizing system [8-9]. In this research, the determination of license plate location is studied through direct usage of Sobel edge detector followed by filling connected components with holes and linear dilation. The earlier processing step is considered to convert an image into a binary image by applying a particular value threshold [10]. This method produces acceptable results when captured license plate image is subjected to high intensity of light or when the vehicle and license plate area have approximately similar colors. Various factors such as camera quality, distance, and weather affect the quality and precision of algorithm performance discussed in this paper [11]. The proposed algorithm is robust to these issues with an acceptable level of accuracy. However, the segmentation stage, including dilation and erosion, imposes several deformity issues in the characters.

Moreover, one critical issue regarding license plate recognition is manipulating the original font style and size, making it challenging to recognize characters [12-15]. The proposed algorithm develops a segmentation section with some unique features to achieve efficient results. Here the camera collects the data as digitized vehicle images, and MATLAB 2020 software is used to process the image to obtain the license plate characters. Applications of the proposed system can be seen in the following table [16-18].

\section{OBSTACLES IN THE DEVELOPMENT OF THE SYSTEM}

geographical, political, economic, cultural, and social conditions, technologies, laws (for example, in the case of the license plate design), and drawing a custom

Table 1. The proposed system applications

Applications in Smart Cities and ITS
(in brief)


script in the region and country in which the person lives are factors that affect the level of algorithm complexity [16]. In the following, we will review and explain those:

- The Impact of climatic and geographical conditions.

- The effect of the terms and conditions regarding the appearance of license plates.

- Impact of climatic and geographical conditions.

\subsection{Impact of climatic and geographical conditions}

In recognizing visual patterns using Image processing methods, the condition and degree of image quality significantly impact the result and the accuracy and speed of processing. There is no doubt that one of the influential parameters in the quality of recorded images is atmospheric factors and Environment, such as the amount of ambient light and the angle of light radiation. Also, factors such as fog, humidity, and dust directly affect image quality [17].

\subsection{The effect of the terms and conditions regarding the appearance of li- cense plates}

The most influential factor in the vehicle's license plate recognition system is the typical design and format of the plate. The country or region of the study is the primary source of information about the license plate. Plate features such as character ratio, character distribution, font type, license plate color, license plate size, size of characters in the license plate, size of the

Spaces between the characters and the total number of characters in the license plate were advantageous for an efficient and practical system. Therefore, using this information will lead to system problems [19-21].

\subsection{The effect of conventional calligraphy}

The letter recognition stage depends more than any other factor on the type of calligraphy used on the license plate. Iranian license plates use specific calligraphy, which itself causes many problems [22-24]. Electronic recognition of printed letters in license plates with Persian characters is tricky and complex as recognizing plates with Latin characters. Recognizing separate letters in the Persian language alone is a massive problem because these letters have different lengths, widths, shapes, and distributions [25-27]. Also, considering this content that the points are separate from the rest of the body and due to the small size of the points, in the noise separation stage, points will be removed with noises, thus making it difficult to recognize the letters. On the other hand, there are considerable similarities Between Persian letters.

\section{IMPLEMENTATION OF ALGORITHM}

Image processing techniques are used to convert the raw data obtained by the cameras into helpful information. This algorithm can be broadly classified into eight stages which can be discussed as follow; mentioned steps have been shown in Fig. (1). Initially, the input color photos are turned to grayscale to perform processing faster on them. The following figure is an example of an input grayscale image shown in Fig. (2). In the next steps, the license plate characters will be extracted from it. 


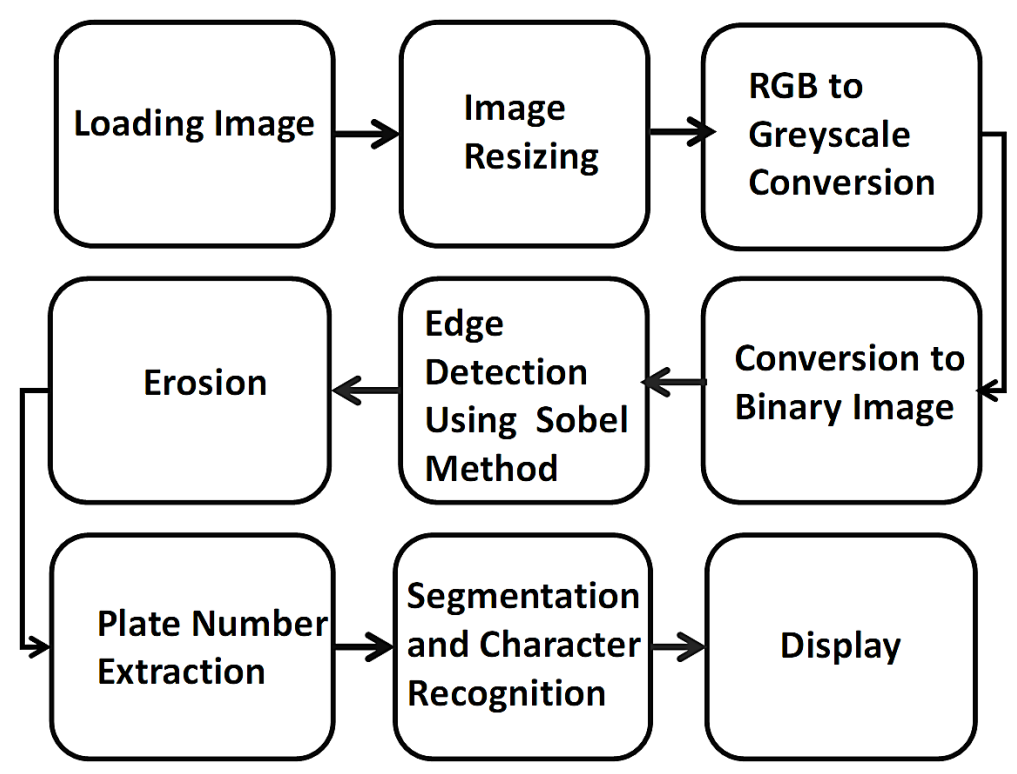

Figure 1. Proposed algorithm's main design stages block-diagram.
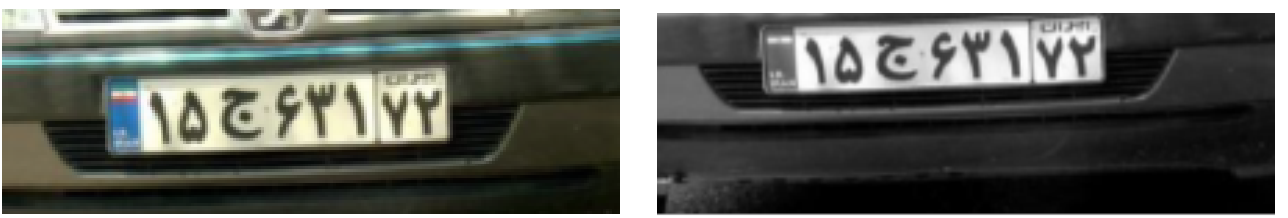

Figure 2. Transforming a color image into grayscale.

\subsection{Pre-processing}

After converting the image to grayscale, the input image will be improved by removing the possible noises. The Gaussian function provides us with a suitable filter for this purpose, according to Eq. (1).

$$
W(x, y)=\frac{1}{2 \pi \sigma^{2}} \mathrm{e}^{\frac{x^{2}+y^{2}}{2 \sigma^{2}}}
$$

\subsection{Edge detection in the image}

For example, there was a sudden decrease in the image's brightness when a fast change from black to white happened, so we have an edge. The numbers and letters were written on the plate have many vertical edges to find their place in the image. Various methods and algorithms exist for finding the edge in image processing. Meanwhile, the Sobel operator is more efficient due to its high speed and low processing volume than other methods. It has a desirable edge detection ability on the images and has two vertical and horizontal edge detection masks. Because numbers and letters have many vertical edges, only the vertical mask is used if the input image does not rotate too much; the rotation effects in the image can be compensated up to about forty-five degrees. Image edging is included as a function in MATLAB software, but before that, this paper has used the statistical features of the image to convert it to binary format. Then the edge finding operation will be performed. As it can be seen, this is far better than performing edge-finding operations on the raw image, which is on a grayscale, Fig. (3). The threshold is determined by calculating the mean and standard variance of the image. The threshold is equal to the sum of the variance and the mean of the image. Then the levels of the image that are greater than this threshold, we attribute the intensity value of one and the other values 
to zero intensity based on Eq. (2), Eq. (3).

$$
\begin{aligned}
T & =P_{m}-P_{\sigma}, \\
p_{m} & =\frac{1}{W} \sum_{i=1}^{W} P_{i} .
\end{aligned}
$$

In the following equations, $P_{i}$ is the number of image pixels, $P_{\sigma}$ is standard variance, $P_{m}$ is the image mean value, $T$ is desired threshold, $B[i, j]$ is the binary image. The depicted matrix is a Sobel mask for detecting the edges, Eq. (4) and Eq. (5).

$$
\begin{aligned}
p_{m} & =\frac{1}{W} \sum_{i=1}^{W}\left|P_{i}-P_{m}\right|, \\
B[i, j] & =\left\{\begin{array}{l}
0, \text { otherwise } \\
1, T \leq P_{i} .
\end{array}\right.
\end{aligned}
$$
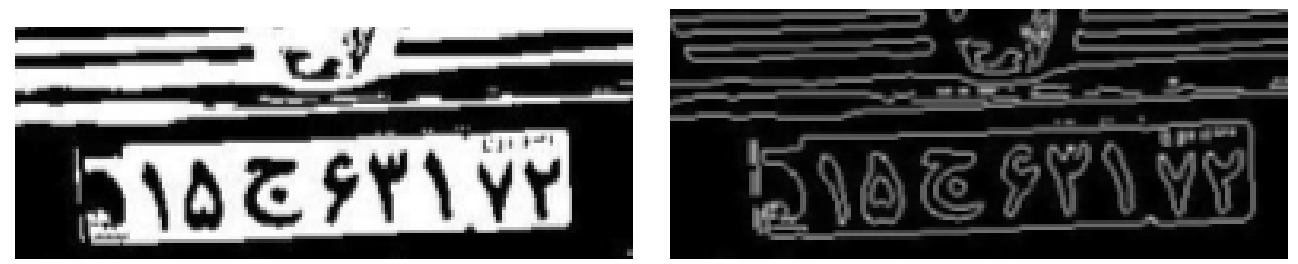

Figure 3. Turning the grayscale into a binary format.

\subsection{Erosion}

Now the erosion operation is performed on the edged image by issuing the following equation. The erosion of $\mathrm{A}$ and $\mathrm{B}$ are defined as the set of all points $\mathrm{X}$ that if $B$ shifts by $x$ again, it fits perfectly inside $A$, and the space in the image has a thin standard edge Fig. (4). These two areas can be separated by erosion based on Eq. (6).

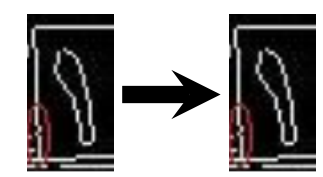

Figure 4. This figure shows erosion operation on the image.

$$
A \oplus B=\left\{x \mid(B)_{x} \subseteq A\right\}
$$

\subsection{Horizontal and vertical image expansion}

After the erosion operation, a horizontal expansion operation is performed on the image. The following equation is defined as eq.3. The expansion of $\mathrm{A}$ with $B$ is the set of all $x^{\prime}$ s that $(\dot{B}) x$ and $A$ have overlap at least in a non-zero element. $(\dot{B}) x$ is the symmetry of $B$, which is around its center, then transfers this symmetry to $x$. Expansion fills the space between the two edges based on Eq. (7). For example, if some discontinuities exist in a straight line in the image. You can see the expansion of the image below, Fig. (5) and Procedure (1).

$$
A \oplus B=\left\{x \mid\left[(\hat{B})_{x} \cap A\right] \subseteq A\right\}
$$




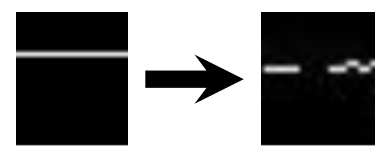

Figure 5. This figure shows the expansion operation on the selected part of the image.

Procedure 1. Expansion procedure of the selected part of the image

pd=imdilate (ps, strel('diamond' , 1));

pe=imerode $(p d, \operatorname{strel}($ 'diamond', 1$))$;

\subsection{Hole filling operation}

A hole is a black dot in a white space in an edged image. Possible holes are filled because the edged image is interconnected at the plate. The image below results from the simultaneous application of these three methods to improve the edges and holes of the image at this stage of the simulation, Fig. (6) and Procedure (2). This step results in the interconnection of more lines in the image, creating closed spaces. Now lines are closer together, the borders are more tangible, and areas can create solid objects.

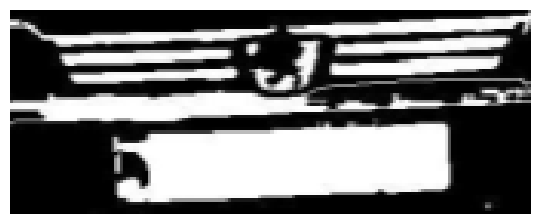

Figure 6. This figure shows an improved image after holes filling operation.

Procedure 2. Holes filling operation

pl=imfill(pe, 'holes');

\section{LICENSE PLATE LOCATION FINDING}

\subsection{Image labeling}

The main feature extracted from the image are the closed spaces can be seen in the following figure. Every white space inside the image is a closed space. We create a structure in which all these closed spaces are numbered sequentially. Then, we enclose each small, labeled area inside a rectangle, which helps for spaces with an irregular structure such as a sloping plate or a rotating coordinate. Find a place and find the degree of tilt or rotation of the license plate would be the other features of this algorithm. A labeled image for a limited number of spaces can be seen below, Fig. (7) and Procedure (3) represent a general view of the performed process to reach the goal. In general, the process of labeling is very important to be done accurately.

\subsection{Selection of license plate location}

Among the spaces created, the space, including the license plate, has the largest area in most cases. This feature is the main point in image locating, so apply this method to the input image; as we expected, it includes the correct location of the license plate, Fig. (8) and Procedure (4). 


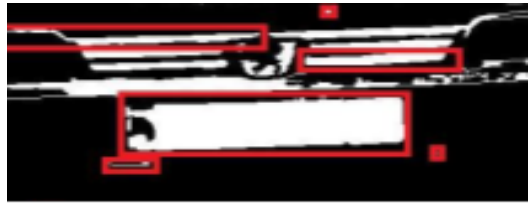

Figure 7. This figure illustrates the labeling operation on the acquired image and the selection of various areas on the image.

Procedure 3. Labeling operation on the acquired image and selection of various areas on the image

$\mathrm{pll}=\mathrm{bwlabel}(\mathrm{pl})$;

stat=regionprops (pll, 'Area' , 'BoundingBox' ,

'Image' , 'Orientation', 'Centroid');

Procedure 4. Location finding operation to select the potential place of license plate

index $=($ find $([$ stat. Area $]==\max ([$ stat.Area $])))$;

The space containing the license plate has an irregular shape, the coordinates of which are found in the image give wrong results. However, enclosing it in a rectangular box with four fixed points of the corners is the method investigated in this paper to find the exact location of the license plate, Fig. (9) and Procedure (5).

Then we improve the image of the plate in terms of contrast and turn it into the grayscale or binary surface, for which the defined threshold in the article can be reused, Fig. (10) and Procedure (6).

\subsection{Tilt and rotation of the plate improvement}

Now we have the binary and raw image of the license plate and use the feature of putting the pixels together in the digital image and finding the inclination angle. In a binary image without distortion, to go from one corner of the image to another, a path must be included pixels connected from only one corner. If the same assumed image moves parallel to one of the sides, we see that the pixels are completely stuck together in a row. For example, look at how the pixels in these two images are arranged along the red line, Figs. (11-12)

Notice that how the two pixels in the zoomed image are placed side by side; they can be the same as the original diameter of the ellipse and can be considered to determine the angle of the actual diameter with the horizontal axis of the image rotation field, Fig. (13), Fig. (14) and Procedure (7).

\subsection{Extracting the license plate characters}

At this stage, we have the license plate image improved and balanced without noise as input. We find the license plate image complement so that the characters

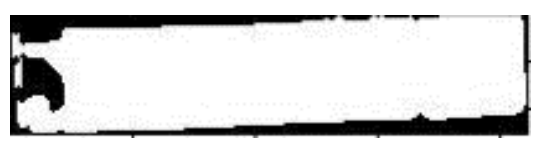

Figure 8. This image illustrates the location finding operation to select the potential place of the license plate. 


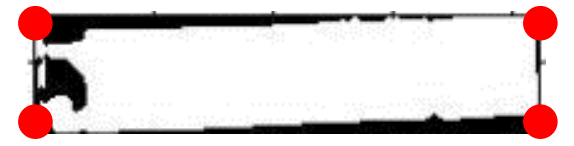

Figure 9. This figure shows the corners selection operation of the potential location of license plate.

Procedure 5. Corners selection operation of the potential location of license plate
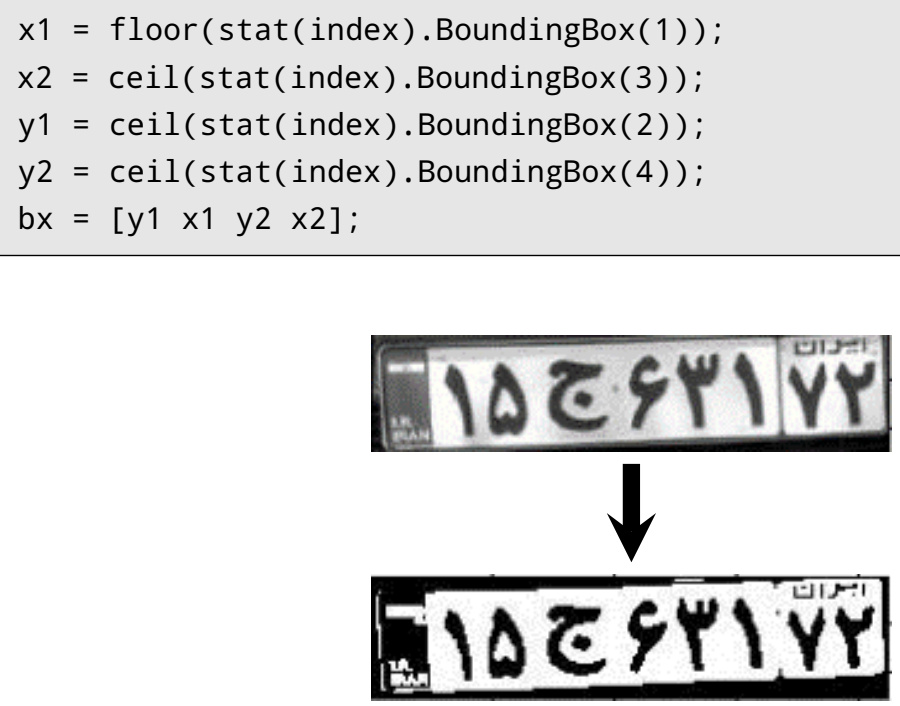

Figure 10. This figure illustrates the improvement operation of a license plate image.

Procedure 6. Improvement operation of a license plate image
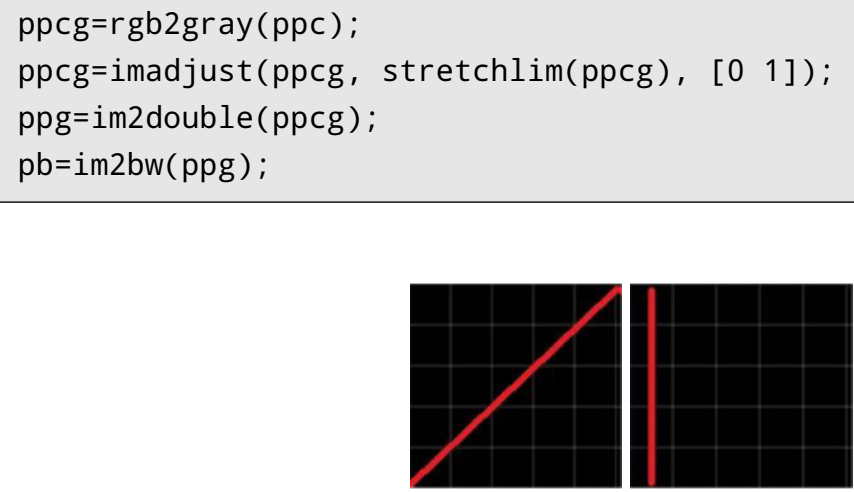

Figure 11. This figure demonstrates the pixels' organization of the acquired image.

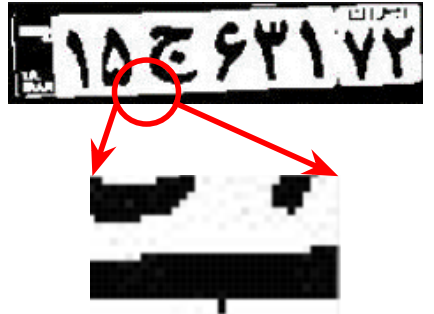

Figure 12. A zoomed image of the license plate before tilt and rotation improvement.

inside the license plate can be seen as one or white intensity. Using a labeled binary image, we start to number the spaces created inside the plate image. In this case, each of the characters in the image forms a closed area which is 


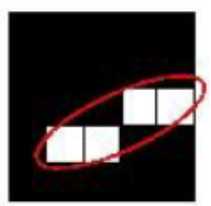

Figure 13. This figure shows the rotation determination operation on the obtained image from the previous stage.

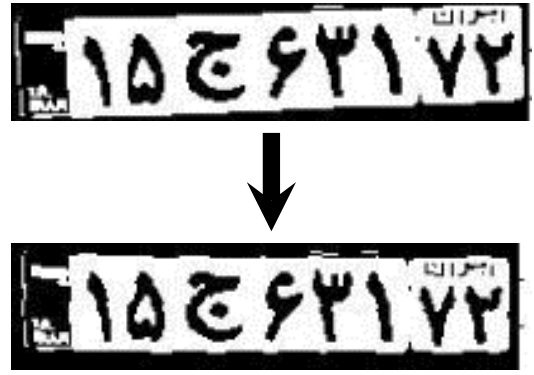

Figure 14. This image shows the license plate after removing the rotation.

Procedure 7. Rotation determination and removing operation

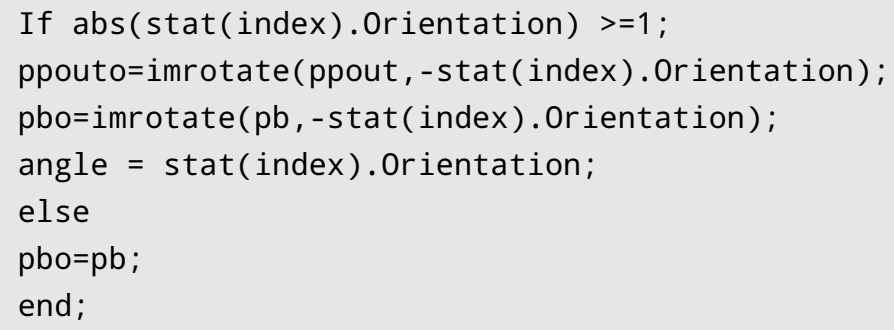

possible to call the label number in order, Fig. (15) and Procedure (8).

\subsection{Characters identification}

We are now in the final stage of the paper. Various methods have been proposed to identify license plates such as neural networks, images correlation functions, similarity-based methods. This paper has been introduced a function called "normal factor" by specifying the characterization of the character's image, Eq. (8) and Procedure (9). Its working principle is based on this feature and extracted character compared with pre-identified samples, considering the assumption that two similar images of equal weight can be placed on top of each other. When the normal factor of those two images has the maximum possible value, this function is introduced as follows. For example, if we put two similar characters on top of each other, the white pixels are more than two different characters, Fig. (16).

$$
N F=\frac{\sum_{i=0}^{m} \sum_{j=0}^{n} T_{i, j} M_{i, j}}{\sum_{i=0}^{m} \sum_{j=0}^{n} T_{i, j}}
$$

$T_{i, j}$ is the weights of pre-identified sample characters and models; $M_{i, j}$ is the binary image of characters extracted from the plate where $N F$ will be between 0 and 1 


\section{DPCSFIVI \\ Wardivilivi}

Figure 15. This figure depicts the extraction of license plate characters operation.

Procedure 8. Rotation determination and removing operation

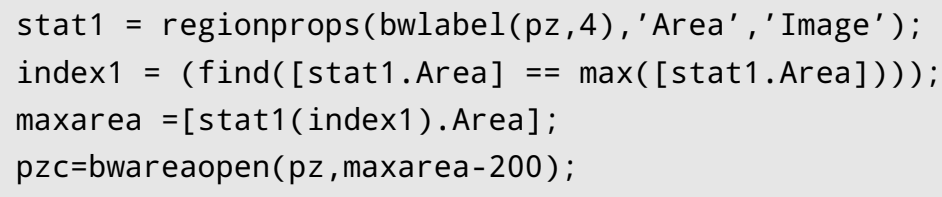

\section{RESULTS' DISCUSSION}

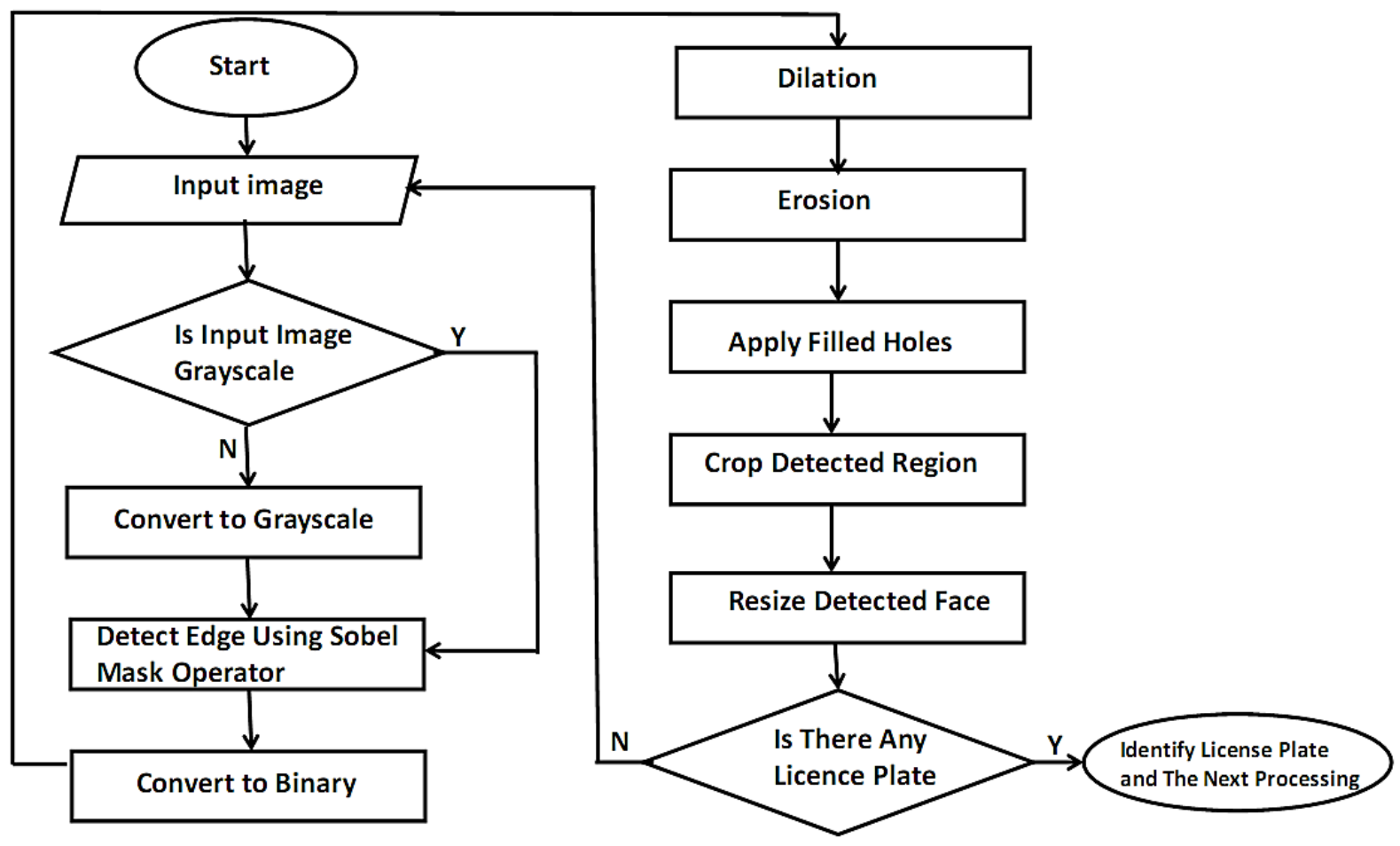

Figure 16. The figure illustrates the proposed algorithm flowchart.

Most of the simulated features presented in this project are more optimized and efficient than other standard algorithms. Several apparent advantages of this method related to others are its incredible speed. In terms of noise, this algorithm is invulnerable to the noises such as low light, plate rotation, and low quality of the image. However, it seems vulnerable to plate contamination and physical damage to the car's license plate. Deleting the threshold setting step in the algorithm is studied to show the power of the algorithm; we see that by deleting this part of the article, the results of the wrong license plate location, Fig. (17). 
Procedure 9. Character's identification operation
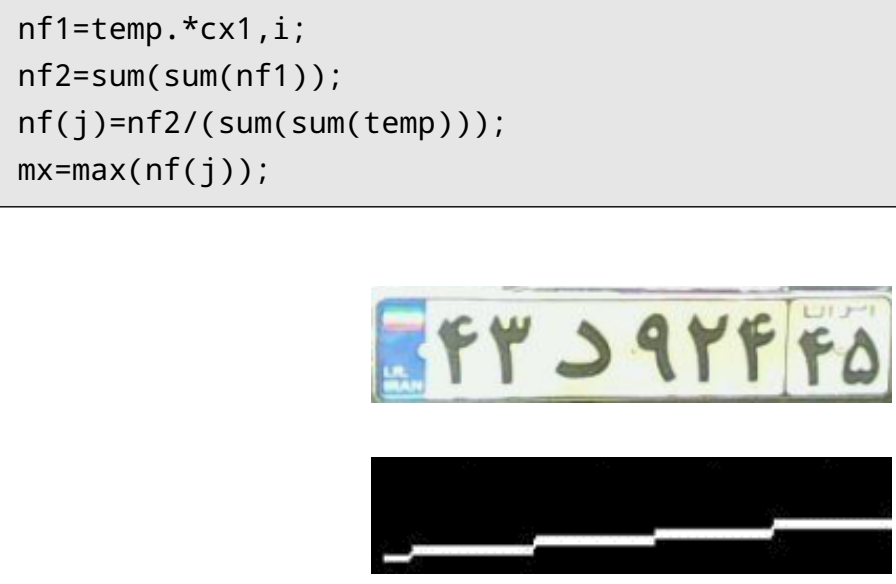

(a)

\section{FY S PYFFA}

(b)

Figure 17. Images (a) and (b) shows license plate positioning without and with threshold setting, respectively.
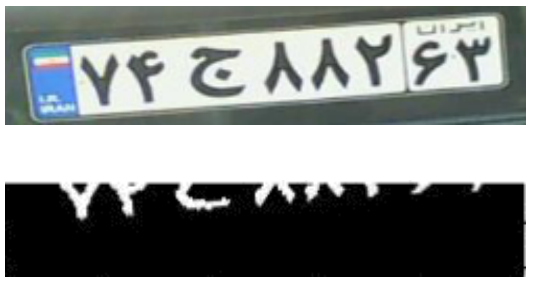

(a)

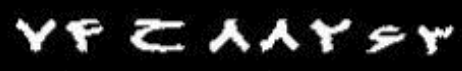

(b)

Figure 18. Images (a) and (b) show license plate positioning without and with rotation improvement.
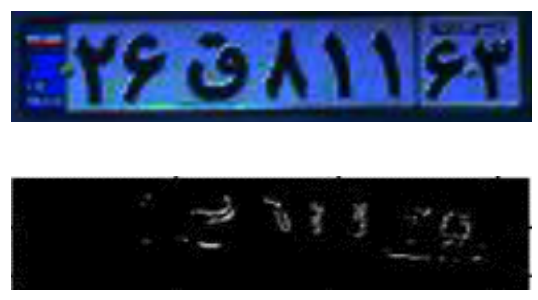

(a)

\section{YoATI}

(b)

Figure 19. Images (a) and (b) show license plate positioning without and with proposed algorithm features in low light, respectively.

To show another plus point of the proposed algorithm, the comparison of the car license plate deflection result with an algorithm without simulated paper features is studied, Fig. (18). Furthermore, comparing low light conditions, we 
see that the algorithm is still robust to the noisy conditions, Fig. (19). A total of 98 images were processed through MATLAB 2020 software. These images were taken in different illumination conditions. Images were obtained from various cameras from $5 \mathrm{MP}$ to $13 \mathrm{MP}$ clarity. Distance varied from 1 meter to 20 meters. Images of vehicles in various weather conditions were processed. The results after processing are presented in Table 2, Below.

Table 2. Tests results of the proposed algorithm.

\begin{tabular}{ccc}
\hline Units & Correct operations & Percentage of accuracy \\
\hline Extraction & $92 / 98$ & 93.87 \\
Segmentation & $95 / 98$ & 96.93 \\
Recognition & $91 / 98$ & 92.85 \\
\hline
\end{tabular}

\section{CONCLUSION}

The proposed method efficiency in this paper can be improved through future development. By increasing resistance to unwanted interference and providing a more comprehensive database of different characters, you can read the license plate characters in the identification stage with a higher level of accuracy. Instead of working on the grayscale image, you can perform operations on color images. Then it would be possible for international license plates to be recognized, and the type of flag on the license plate and its nationality can be extracted. A graphical interface can be generated for the algorithm to be used by the user without the need to know the program's technical features. It can be realized by using the Matlab GUI toolbox.

\section{Conflicts of Interest}

The authors declare that they have no conflicts of interest.

\section{Acknowledgement}

This work was supported without any funding.

\section{References}

[1] Kim, S. K., Kim, D. W., \& Kim, H. J. (1996, September). A recognition of vehicle license plate using a genetic algorithm based segmentation. In Image Processing, 1996. Proceedings., International Conference on (Vol. 2, pp. 661-664). IEEE.

[2] Arth, C., Limberger, F., \& Bischof, H. (2007, June). Real-time license plate recognition on an embedded DSP-platform. In Computer Vision and Pattern Recognition, 2007. CVPR'07. IEEE Conference on (pp. 1-8). IEEE.

[3] Ozbay, S., \& Ercelebi, E. (2005). Automatic vehicle identification by plate recognition. World Academy of Science, Engineering and Technology, 9(41), 222-225.

[4] Sulehria, H. K., Ye Zhang, D. I., \& Sulehria, A. K. (2008). Vehicle number plate recognition using mathematical morphology and neural networks. WSEAS Transactions on Computers, 7.

[5] Qadri, M. T., \& Asif, M. (2009, April). Automatic number plate recognition system for vehicle identification using optical character recognition. In Education Technology and Computer, 2009. ICETC'09. International Conference on (pp. 335-338). IEEE.

[6] Roy, A., \& Ghoshal, D. P. (2011, March). Number Plate Recognition for use in different countries using an improved segmentation. In Emerging Trends and Applications in Computer Science (NCETACS), 2011 2nd National Conference on (pp. 1-5). IEEE. 
[7] Arulmozhi, K., Perumal, S. A., Priyadarsini, C. T., \& Nallaperumal, K. (2012, December). Image refinement using skew angle detection and correction for Indian license plates. In Computational Intelligence \& Computing Research (ICCIC), 2012 IEEE International Conference on (pp. 1-4). IEEE.

[8] An Efficient Parallel Genetic Algorithm Solution for Vehicle Routing Problem in Cloud Implementation of the Intelligent Transportation Systems M Abbasi, M Rafiee, MR Khosravi, A Jolfaei, VG Menon, JM Koushyar Journal of Cloud Computing, 2020.

[9] Enhancing the Performance of Flow Classification in SDN-based Intelligent Vehicular Networks M Abbasi, H Rezaei, VG Menon, L Qi, MR Khosravi IEEE Transactions on Intelligent Transportation Systems 22 (7), 4141-4150, 2021.

[10] C Chen, Y Zhang, MR Khosravi, Q Pei, S Wan, An Intelligent Platooning Algorithm for Sustainable Transportation Systems in Smart Cities, IEEE Sensors Journal 21 (14), 15437-15447, 2021.

[11] M Abbasi, A Najafi, M Rafiee, MR Khosravi, VG Menon, G Muhammad, Efficient Flow Processing in 5G-Envisioned SDN-Based Internet of Vehicles Using GPUs, IEEE Transactions on Intelligent Transportation Systems, 2020.

[12] M Abbasi, M Yaghoobikia, M Rafiee, A Jolfaei, MR Khosravi, Energy-efficient Workload Allocation in Fog-Cloud based Services of Intelligent Transportation Systems Using a Learning Classifier System, IET Intelligent Transport Systems, 2020.

[13] M Abbasi, M Yaghoobikia, M Rafiee, MR Khosravi, VG Menon, Optimal Distribution of Workloads in Cloud-Fog Architecture in Intelligent Vehicular Networks, IEEE Transactions on Intelligent Transportation Systems 22 (7), 4706-4750

[14] Raskar, R. R., \& Dabhade, R. G. (2015). Automatic Number Plate Recognition (ANPR). International Journal of Emerging Technology and Advanced Engineering, 5 .

[15] García-Sánchez, S., Aubert, S., Iraqui, I., Janbon, G., Ghigo, J. M., \& d’Enfert, C. (2004). Candida albicans biofilms: a developmental state associated with specific and stable gene expression patterns. Eukaryotic cell, 3(2), 536-545.

[16] Dewan, S., Bajaj, S., \& Prakash, S. (2015, June). Using Ant's Colony Algorithm for improved segmentation for number plate recognition. In Computer and Information Science (ICIS), 2015 IEEE/ACIS 14th International Conference on (pp. 313-318). IEEE.

[17] Kapoor, A., Bhat, S. I., Shidnal, S., \& Mehra, A. (2016, October). Implementation of IoT (Internet of Things) and Image processing in smart agriculture. In Computation System and Information Technology for Sustainable Solutions (CSITSS), International Conference on (pp. 21-26). IEEE.

[18] Khan, J. A., \& Shah, M. A. (2016, September). Car Number Plate Recognition (CNPR) system using multiple template matching. In Automation and Computing (ICAC), 2016 22nd International Conference on (pp. 290-295). IEEE.

[19] Babu, K. M., \& Raghunadh, M. V. (2016, May). Vehicle number plate detection and recognition using bounding box method. In Advanced Communication Control and Computing Technologies (ICACCCT), 2016 International Conference on (pp. 106-110). IEEE.

[20] Agarwal, A., \& Goswami, S. (2016, February). An Efficient Algorithm for Automatic Car Plate Detection \& Recognition. In Computational Intelligence \& Communication Technology (CICT), 2016 Second International Conference on (pp. 644-648). IEEE.

[21] Osslan Osiris Vergara Villegas, Daniel Gonzalez Balderrama, Humberto de Jesus Ochoa Dominguez, and Vianey Guadalupe Cruz Sanchez, "License Plate Recognition Using A Novel Fuzzy Multilayer NeuralNetwork", International Journal of Computers, Issue.1, vol. 3, pp. 31—40, 2009. 
[22] R. Gonzalez, R.Woods, S. Eddins, "Digital Image Processing Using MATLAB ${ }^{\circledR}$, ISBN 978-81-7758-898-9, Pearson Education, South Asia, 2009.

[23] Ch. Jaya Lakshmi, Dr. A. Jhansi Rani, Dr. K. Sri Ramakrishna and M. KantiKiran, "A Novel Approach for Indian License Plate Recognition System", (IJAEST) International Journal of Advanced Engineering Sciences And Technologies, Vol. - 6, Issue No. 1, 010-014.

[24] Satadal Saha, Subhadip Basu and Mita Nasipuri, "Automatic Localization and Recognition of License Plate Characters for Indian Vehicles", International Journal Computer Science Emerging Tech, August 2011.

[25] Ali Tahir, Hafiz Adnan Habib and M. Fahad Khan, "License Plate Recognition Algorithm for Pakistani License Plates", Canadian Journal on Image Processing and Computer Vision, April 2010.

[26] H. Swethalakshmi, et al., "Online Handwritten Character Recognition of Devanagari and Telugu Characters using Support Vector Machines," Guy Lorette. Tenth International Workshop on Frontiers in Handwriting Recognition, La Baule (France), Suvisoft, pp. 1-6, 2006.

[27] A. R. Syafeeza, et al., "Convolutional Neural Networks with Fused Layers Applied to Face Recognition," International Journal of Computational Intelligence and Applications, vol/issue: 14(3), 2015.

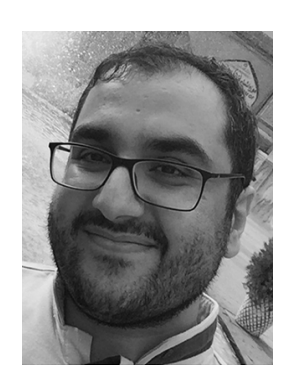

Mohammad R. Khosravi received the B.Sc., M.Sc., and Ph.D. degrees in electrical engineering with expertise in communications and signal processing. He is currently with the Department of Electrical and Electronic Engineering Engineering, Shiraz, Iran. His main research interests include optical and radar imaging, digital image processing, remote sensing systems, computer communications, cyber-physical systems, internet of things, and industrial sensor networks.

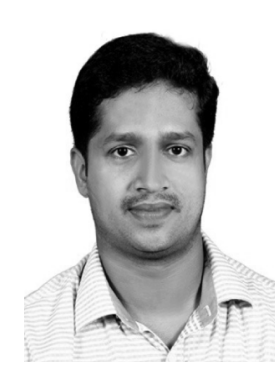

Linss T. Alex received the degree in electrical and electronics engineering from Mahatma Gandhi University, India, in 2007, the master's degree in power electronics from Amrita Vishwa Vidyapeetham University, India, in 2011, and the Ph.D. degree in electrical and electronics engineering from the SRM Institute of Science and Technology, Chennai, India, in 2020. He is currently working as an Associate Professor with the Department of Electrical and Electronics Engineering, Met's School of Engineering, A. P. J. Abdul Kalam Technological University, Kerala, India. His research interests include renewable energy systems, the Internet of Things, artificial intelligence, and electric vehicles. 